\title{
Elementary Proofs for Convolution Identities of Abel and Hagen-Rothe
}

\author{
Wenchang $\mathrm{Chu}^{*}$ \\ Hangzhou Normal University \\ Institute of Combinatorial Mathematics \\ Hangzhou 310036, P. R. China
}

Submitted: Feb 25, 2010; Accepted: Apr 20, 2010; Published: Apr 30, 2010

Mathematics Subject Classifications: 05A10, 05A19

\begin{abstract}
By means of series-rearrangements and finite differences, elementary proofs are presented for the well-known convolution identities of Abel and Hagen-Rothe.
\end{abstract}

\section{Introduction}

There are numerous identities in mathematical literature. Among them, Newton's binomial theorem is well-known

$$
\sum_{k=0}^{n}\left(\begin{array}{l}
n \\
k
\end{array}\right) x^{k} y^{n-k}=(x+y)^{n} .
$$

Abel found its following deep generalization (cf. Comtet [6, §3.1] for example)

$$
\sum_{k=0}^{n} \frac{a}{a+b k} \frac{(a+b k)^{k}}{k !} \frac{(c-b k)^{n-k}}{(n-k) !}=\frac{(a+c)^{n}}{n !} .
$$

Another binomial identity is the Chu-Vandermonde convolution formula

$$
\sum_{k=0}^{n}\left(\begin{array}{l}
x \\
k
\end{array}\right)\left(\begin{array}{c}
y \\
n-k
\end{array}\right)=\left(\begin{array}{c}
x+y \\
n
\end{array}\right) .
$$

\footnotetext{
*Email address: chu. wenchang@unisalento.it
} 
It has been generalized by Hagen and Rothe to the following one (cf. Chu [3,4], Gould [8] and Graham et al $[10, \S 5.4])$

$$
\sum_{k=0}^{n} \frac{a}{a+b k}\left(\begin{array}{c}
a+b k \\
k
\end{array}\right)\left(\begin{array}{c}
c-b k \\
n-k
\end{array}\right)=\left(\begin{array}{c}
a+c \\
n
\end{array}\right) .
$$

These convolution identities are fundamental in enumerative combinatorics. The reader can refer to Strehl [15] for a historical note. The existing proofs for the identities of Abel and Hagen-Rothe can be summarized as follows:

- The classical Lagrange expansion formula: Riordan [13, §4.5].

- Gould-Hsu Inverse series relations: Chu and Hsu $[1,5]$.

- Generating function method: Gould [8,9] (see Chu [2] also).

- The Cauchy residue method of integral representation: Egorychev [7, §2.1].

- Lattice path combinatorics: Mohanty [11, §4.2] and Narayana [12, Appendix].

- Riordan arrays (which can trace back to Lagrange expansion): Sprugnoli [14].

However to our knowledge, there does not seem to have appeared really elementary proofs for these identities in classical combinatorics, even though this has long been desirable. By utilizing the standard method of series-rearrangement that was systematically used by Wilf [16], this short paper will present elementary proofs for the convolution identities of Abel and Hagen-Rothe. It may be unexpected that these proofs are surprisingly simple, which depend upon the following almost trivial fact that the finite differences of a polynomial results in zero if the polynomial degree is less than the order of differences.

\section{Proofs of the Abel Formulae}

According to the binomial theorem, we have

$$
(c-b k)^{n-k}=\sum_{i=k}^{n}(-1)^{i-k}\left(\begin{array}{c}
n-k \\
i-k
\end{array}\right)(a+c)^{n-i}(a+b k)^{i-k} .
$$

Consider the following double sum

$$
\begin{aligned}
U & :=\sum_{k=0}^{n} \frac{a}{a+b k} \frac{(a+b k)^{k}}{k !} \frac{(c-b k)^{n-k}}{(n-k) !} \\
& =\frac{a}{n !} \sum_{k=0}^{n}\left(\begin{array}{l}
n \\
k
\end{array}\right)(a+b k)^{k-1} \sum_{i=k}^{n}(-1)^{i-k}\left(\begin{array}{c}
n-k \\
i-k
\end{array}\right)(a+c)^{n-i}(a+b k)^{i-k} .
\end{aligned}
$$


Interchanging the summation order and observing that

$$
\left(\begin{array}{l}
n \\
k
\end{array}\right)\left(\begin{array}{c}
n-k \\
i-k
\end{array}\right)=\left(\begin{array}{l}
n \\
i
\end{array}\right)\left(\begin{array}{l}
i \\
k
\end{array}\right)
$$

we get the following expression

$$
U=\frac{a}{n !} \sum_{i=0}^{n}\left(\begin{array}{l}
n \\
i
\end{array}\right)(a+c)^{n-i} \sum_{k=0}^{i}(-1)^{i-k}\left(\begin{array}{l}
i \\
k
\end{array}\right)(a+b k)^{i-1} .
$$

When $i>0$, the inner sum with respect to $k$ vanishes because it results in the $i$ th differences of the polynomial $(a+b x)^{i-1}$ of degree $i-1$. Therefore we have found that $U=\frac{(a+c)^{n}}{n !}$, which confirms exactly $(1)$.

\section{Proofs of Hagen-Rothe Identities}

Analogously we have from the $\mathrm{Chu}$-Vandermonde convolution

$$
\left(\begin{array}{c}
c-b k \\
n-k
\end{array}\right)=\sum_{i=k}^{n}\left(\begin{array}{c}
a+c \\
n-i
\end{array}\right)\left(\begin{array}{c}
-a-b k \\
i-k
\end{array}\right)
$$

Then consider another double sum

$$
\begin{aligned}
V & :=\sum_{k=0}^{n} \frac{a}{a+b k}\left(\begin{array}{c}
a+b k \\
k
\end{array}\right)\left(\begin{array}{c}
c-b k \\
n-k
\end{array}\right) \\
& =\sum_{k=0}^{n} \frac{a}{a+b k}\left(\begin{array}{c}
a+b k \\
k
\end{array}\right) \sum_{i=k}^{n}\left(\begin{array}{c}
a+c \\
n-i
\end{array}\right)\left(\begin{array}{c}
-a-b k \\
i-k
\end{array}\right) .
\end{aligned}
$$

Interchanging the summation order and observing that

$$
\frac{a}{a+b k}\left(\begin{array}{c}
a+b k \\
k
\end{array}\right)\left(\begin{array}{c}
-a-b k \\
i-k
\end{array}\right)=\frac{(-1)^{i-k} a}{a+b k-k+i}\left(\begin{array}{l}
i \\
k
\end{array}\right)\left(\begin{array}{c}
a+b k-k+i \\
i
\end{array}\right)
$$

we get another double sum expression

$$
V=\sum_{i=0}^{n}\left(\begin{array}{l}
a+c \\
n-i
\end{array}\right) \sum_{k=0}^{i}(-1)^{i-k}\left(\begin{array}{l}
i \\
k
\end{array}\right) \frac{a}{a+b k-k+i}\left(\begin{array}{c}
a+b k-k+i \\
i
\end{array}\right) .
$$

When $i>0$, the inner sum with respect to $k$ becomes zero because it results again in the finite differences of a polynomial with the polynomial degree less than the difference order by one. Consequently we have shown that $V=\left(\begin{array}{c}a+c \\ n\end{array}\right)$, which is equivalent to (2). 
For the identities displayed in (1) and (2), their linear combinations yield the following respective symmetric forms

$$
\begin{aligned}
& \sum_{k=0}^{n} \frac{a}{a+b k} \frac{(a+b k)^{k}}{k !} \frac{c-b n}{c-b k} \frac{(c-b k)^{n-k}}{(n-k) !}=\frac{a+c-b n}{a+c} \frac{(a+c)^{n}}{n !} \\
& \sum_{k=0}^{n} \frac{a}{a+b k}\left(\begin{array}{c}
a+b k \\
k
\end{array}\right) \frac{c-b n}{c-b k}\left(\begin{array}{c}
c-b k \\
n-k
\end{array}\right)=\frac{a+c-b n}{a+c}\left(\begin{array}{c}
a+c \\
n
\end{array}\right) .
\end{aligned}
$$

The approach presented here can also be employed to prove them similarly. The details are left to the reader as exercises.

\section{References}

[1] W. Chu, Inversion Techniques and Combinatorial Identities: A quick introduction to hypergeometric evaluations, Math. Appl. 283 (1994), 31-57.

[2] W. Chu, Generating functions and combinatorial identities, Glasnik Matematicki 33 (1998), 1-12.

[3] W. Chu, Binomial convolutions and determinant identities, Discrete Math. 204:1-3 (1999), 129-153.

[4] W. Chu, Some binomial convolution formulas, The Fibonacci Quarterly 40:1 (2002), 19-32.

[5] W. Chu - L. C. Hsu, Some new applications of Gould-Hsu inversions, J. Combinatorics, Information \& System Sciences 14:1 (1990), 1-4.

[6] L. Comtet, Advanced Combinatorics, D. Reidel Publishing company, DordrechtHolland, 1974.

[7] G. P. Egorychev, Integral Representation and the Computation of Combinatorial Sums, Translated from the Russian by H. H. McFadden: Translations of Mathematical Monographs 59; American Mathematical Society, Providence, RI, 1984. x+286pp.

[8] H. W. Gould, Some generalizations of Vandermonde's convolution, Amer. Math. Month. 63:1 (1956), 84-91.

[9] H. W. Gould, New inverse series relations for finite and infinite series with applications, J. Math. Res. \& Expos. 4:2 (1984), 119-130.

[10] R. L. Graham - D. E. Knuth - O. Patashnik, Concrete Mathematics, Addison-Wesley Publ. Company, Reading, Massachusetts, 1989.

[11] S. G. Mohanty, Lattice Path Counting and Applications, Z. W. Birnbaum and E. Lukacs, 1979.

[12] T. V. Narayana, Lattice path combinatorics with statistical applications, University of Toronto Press, Toronto - 1979. 
[13] J. Riordan, Combinatorial Identities, John Wiley \& Sons, 1968.

[14] R. Sprugnoli, Riordan arrays and the Abel-Gould identity, Discrete Math. 142 (1995), 213-233.

[15] V. Strehl, Identities of Rothe-Abel-Schläfli-Hurwitz-type, Discrete Mathematics 99:1-3 (1992), 321-340.

[16] H. S. Wilf, The "snake-oil" method for proving combinatorial identities, Surveys in Combinatorics, 1989 (Norwich, 1989), 208-217.

The Corresponding Address

Dipartimento di Matematica

Università del Salento

Lecce-Arnesano P. O. Box 193

73100 Lecce, Italy

Email chu.wenchang@unisalento.it 